\title{
GEOGRAPHIC MAPS ClassifiCATION BASED ON L*A*B COLOR SYSTEM
}

\author{
Salam m. Ghandour ${ }^{1}$ and Nidal M. Turab ${ }^{2}$ \\ ${ }^{1}$ CIS Dept. University Of Jordan, Amman Jordan \\ ${ }^{2}$ Computer Networks And Information Security Dept. , Al Ahliyya Amman University, \\ Amman- Jordan
}

\begin{abstract}
Today any geographic information system (GIS) layers became vital part of any GIS system, and consequently, the need for developing automatic approaches to extract GIS layers from different image maps like digital maps or satellite images is very important.

Map classification can be defined as an image processing technique which creates thematic maps from scanned paper maps or remotely sensed images. Each resultant theme will represent a GIS layer of the images.

A new proposed approach to extract GIS layers (classes) automatically based on $L * A * B$ colorsystem selected from ( $A$ and $B)$ is proposed in this paper, our experiments shows that the hsi color space gives better than $L^{*} A * B$.
\end{abstract}

\section{KEYWORDS}

L*a*b color space, HSI color space, GIS, GIS layers, classification

\section{INTRODUCTION}

Map classification techniques used to extract layers had been published and studies recently. Classification techniques are classified into: [9].

- Supervised classification: the researcher or user performs the following steps:

- The interesting geographic region to test assumption is specified.

- Carefully classifies the classes of interest.

- Selects asuitableclassification algorithm and then collects initial data used to train the algorithm.

- Selects the characteristicsof the most optimal variables.

- Applies classification algorithm produces to produce map and assigns an unknown pixel to its dominant class.

- Evaluates any errors.

- Unsupervised classification techniques: the researcher or user performs the following steps:

- Identifies how many classes to generate and which bands to use.

- The software clusters the pixels into the set of classes.

- Identifies the classes.

DOI: $10.5121 / \mathrm{ijcnc} .2016 .8306$ 
In this paper, we contribute the $\mathrm{L}^{*} \mathrm{a} * \mathrm{~b}$ color space model to extract GIS layers. This is performed by selecting the $a^{*}$ and $b^{*}$ components of the $L^{*} a^{*} b$ color space model. We then compare our results with HIS color model [1].

The paper is organized as follows. In section 1 is an introduction to classification techniques; related work is summarized in section 2. Our proposed new algorithm for automatic extraction of GIS Layers is explained in section 3. While in section 4 we summarize the results of the experiments that conducted to evaluate the proposed map clustering system. Finally, the paper is concluded in section 5 .

\section{RELATED WORK}

The maximum likelihood algorithm is one of the familiar supervised classification algorithms [8],[10]. The maximum likelihood decision rule is based on calculating the probability of pixels belonging to a predefined set of classes, thenthesepixelsare assigned to the highest probability class. However, this algorithm assumes normally distribution of training data as shown in [5]..

The authors of [3] presented an unsupervised clustering, where the image map data is split,the resulting classification mapis composedof a set of classes. Then theresulting classes are assigned to layers. In [4] the authors studied the integrated spectral information with measures of texture, in the form of the variance and the variogram;theyfound that the accuracy was greater than of maximum likelihood. I $n$ [6] the authors presented an overview of significant advances made in the emerging field of nature-inspired computing (NIC); with a focus on the physics- and biologybased approaches and algorithms. They concluded that the field of nature-inspired computing is large and expanding.

In [10] the authors proposed an Agglomerative Hierarchical Clustering based on High-Resolution Remote Sensing Image Segmentation Algorithm.Their conducted experiment shows that the results of this algorithm are better than the K-Means' and are very close to the artificial extraction results.

\section{Proposed APPROACH}

Our proposed approach for GEOGRAPHIC MAPS CLASSIFICATION BASED ON L*a*b COLOR SYSTEM. . The L*a*b color space is a geometrical color-opponent space which has the shape of a sphere. Its composed of the following ingredients: $\mathrm{L}$ is the lightness, while $\mathrm{a}$ and $\mathrm{b}$ together represent the color and saturation information [7], [11]. Figure 1 illustrates the L*a*b color space.

The $\mathrm{L}$, $\mathrm{a}$, and $\mathrm{b}$ axes provide a practical combination of the orthogonal simplicity of the RGB color space and the other color spaces such as HSI which are used in many color management systems[11].

The L*a*b color space is considered to be "perceptually uniform," which means that any detectable visual difference in the color space can be represented by a constant distance in any direction or location. $L^{*} a * b$ is used widely as a standard space to compare colors [2], [11]. In this paper, we are interested in the ( $\mathrm{a}, \mathrm{b}$ ) component only. 

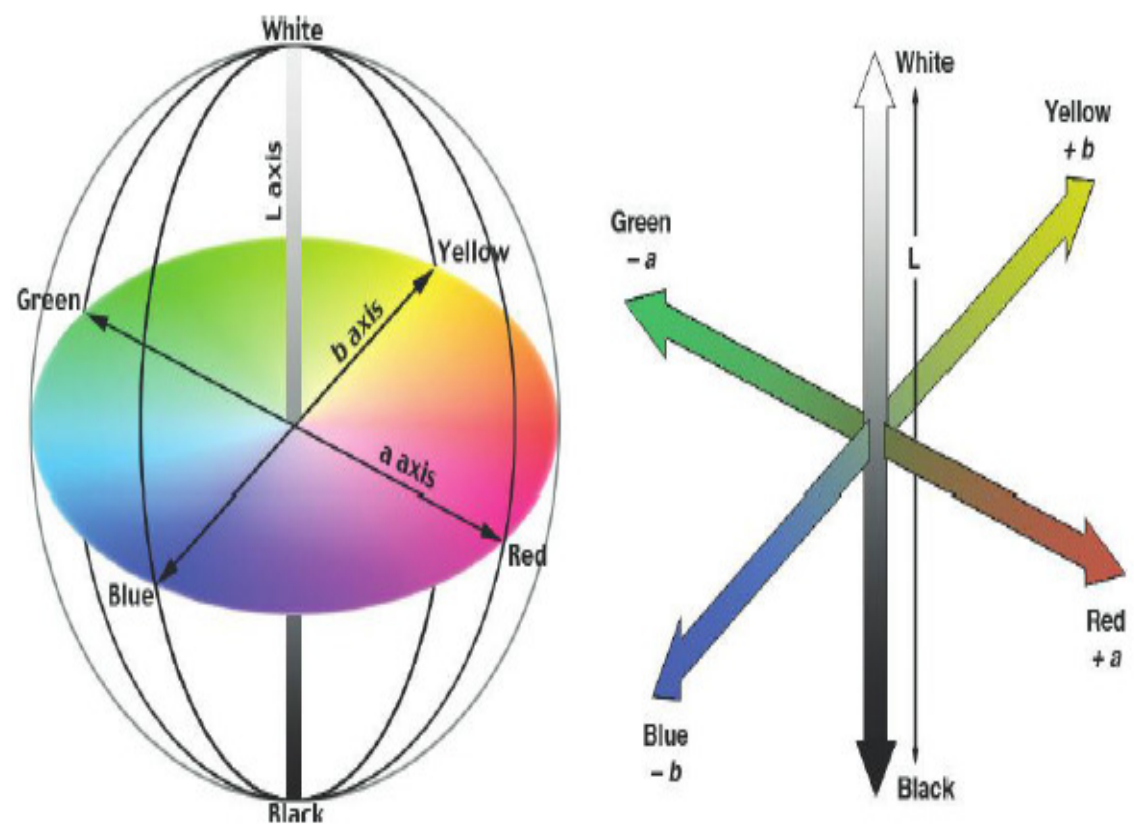

Figure 1. L*a*b color space [2]

Equation (1) represents a mathematical relationship between L, a, b and R, G, B [11]:

$$
\left[\begin{array}{l}
L \\
a \\
b
\end{array}\right]=\left[\begin{array}{ccc}
\frac{1}{3} & \frac{1}{3} & \frac{1}{3} \\
\frac{-\sqrt{2}}{6} & \frac{-\sqrt{2}}{6} & \frac{2 \sqrt{2}}{6} \\
\frac{1}{\sqrt{2}} & \frac{-1}{\sqrt{2}} & 0
\end{array}\right] *\left[\begin{array}{l}
R \\
G \\
B
\end{array}\right]
$$

Equation1. The relationship between $\mathrm{L}, \mathrm{a}, \mathrm{b}$ and $\mathrm{R}, \mathrm{G}, \mathrm{B}$

The proposed map clustering system uses image processing and machine learning techniques. Figure 2 illustrates the flowchart for the proposed system. The first stage after the system start up is to select the input map image. Then, that image is processed for contrast enhancement as a preprocessing stage. At this stage, the image color values distribution is changed to cover a wider range of colors. 
International Journal of Computer Networks \& Communications (IJCNC) Vol.8, No.3, May 2016

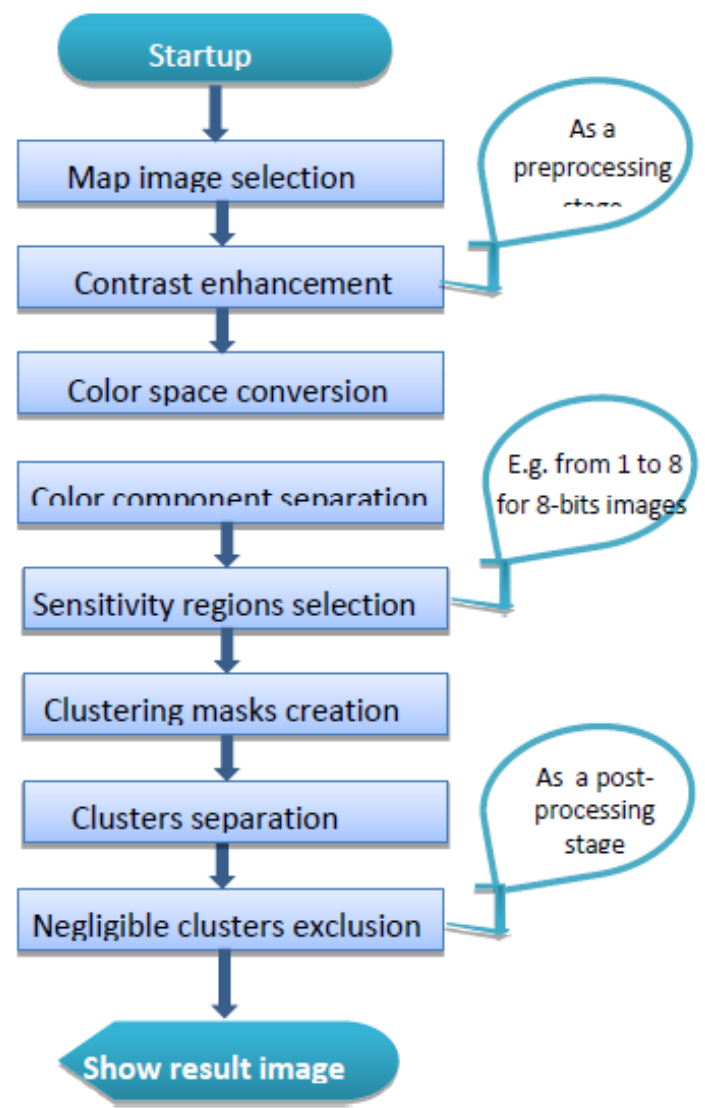

Figure 2. Flowchart of the proposed map clustering system

The enhancement of image contrast yields better clustering results when the clustering is based on colors. Figure 3 shows an example for image contrast enhancement.

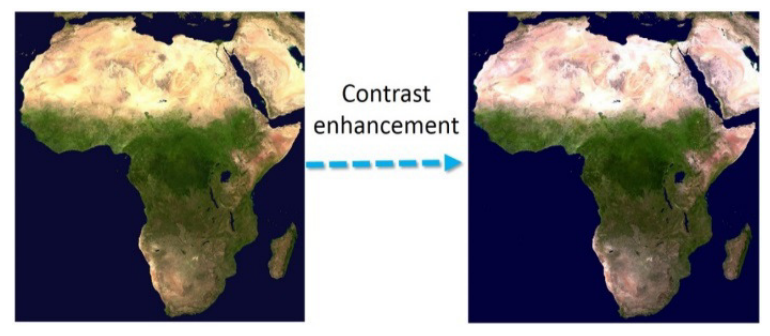

Figure 3. An example of contrast enhancement

Then, the color space used for representing the image is converted to another color space that allowsthe separation of the color components and the light intensity unlike the original RGB color space. This research investigated two color spaces: HSI and $\mathrm{L}^{*} \mathrm{a} * \mathrm{~b}$.

Figure 4 gives an example of the $\mathrm{L}^{*} \mathrm{a} * \mathrm{~b}$ components for an image. The HSI color space gives better clustering results than $\mathrm{L}^{*} \mathrm{a} * \mathrm{~b}$, as will be discussed in detail later. 


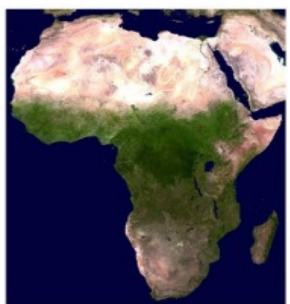

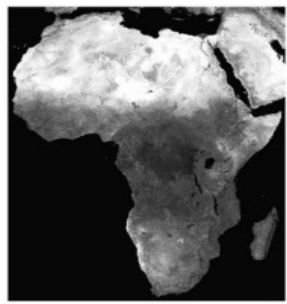

L channel

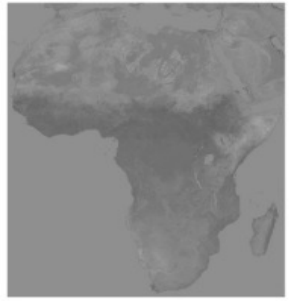

a channel

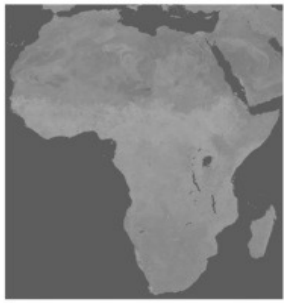

b channel

Figure 4.The $\mathrm{L} * \mathrm{a} * \mathrm{~b}$ components for an image

The system then extracts the color components from the converted image representation and ignores the lightness component.

Finally, the system sensitivity level should be specified by the user so that the system can define the sensitivity regions. Sensitivity level is an integer number that starts from 1 and up to image bit depth (color depth).

The sensitivity level is used to define the number of equally-sized color regions obtained from the HSI or $\mathrm{L}^{*} \mathrm{a}^{*} \mathrm{~b}$ chromaticity distribution, which are called sensitivity regions. To define the chromaticity distribution in HSI color space, a slice of the HSI color space is taken at a fixed intensity value. The same is done in the $\mathrm{L}^{*} \mathrm{a} * \mathrm{~b}$ color space, where a chromaticity distribution is taken at a fixed lightness. Figure 5 shows the chromaticity distribution taken from each color space.

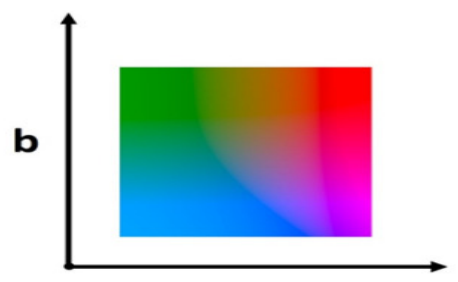

a

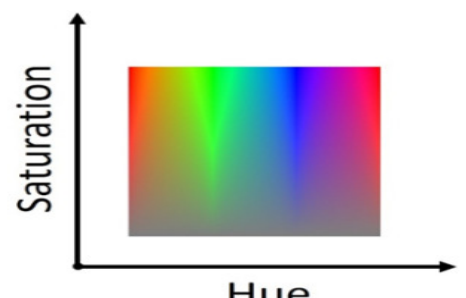

Hue

Figure 5.The chromaticity distribution for: $(\mathrm{a}, \mathrm{b}) \mathrm{L} * \mathrm{a} * \mathrm{~b}$ and HIS

A sensitivity region is in the chromaticity distribution which contains colors to be considered as a cluster. The number of the regions is defined by the equation (2):

Number of sensitivity regions $=2^{1+\text { sensitivity level }}$ 
Figure 6 shows an example of sensitivity regions for $\mathrm{L}^{*} \mathrm{a} * \mathrm{~b}$ color space when the sensitivity level is 3 and it contains 16 sensitivity regions.

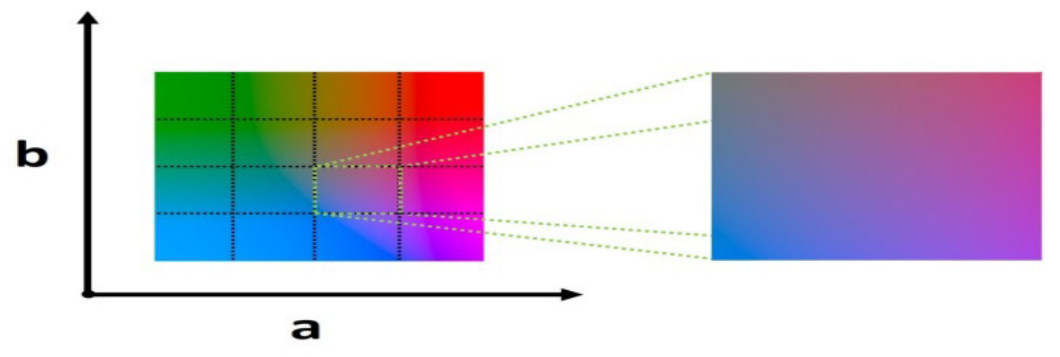

Figure 6.An example of sensitivity regions for $\mathrm{L}^{*} \mathrm{a} * \mathrm{~b}$ color space when the sensitivity level is 3

For each sensitivity region, all the colors in that region are used in a mask to extract the pixels having matching colors from the input map image and the pixels having other colors will be masked as black pixels. After all sensitivity regions are used in the masks, the system will have a number of clusters equal to the number of sensitivity regions. Figure 7 shows the output of masking a sample image with one sensitivity region.

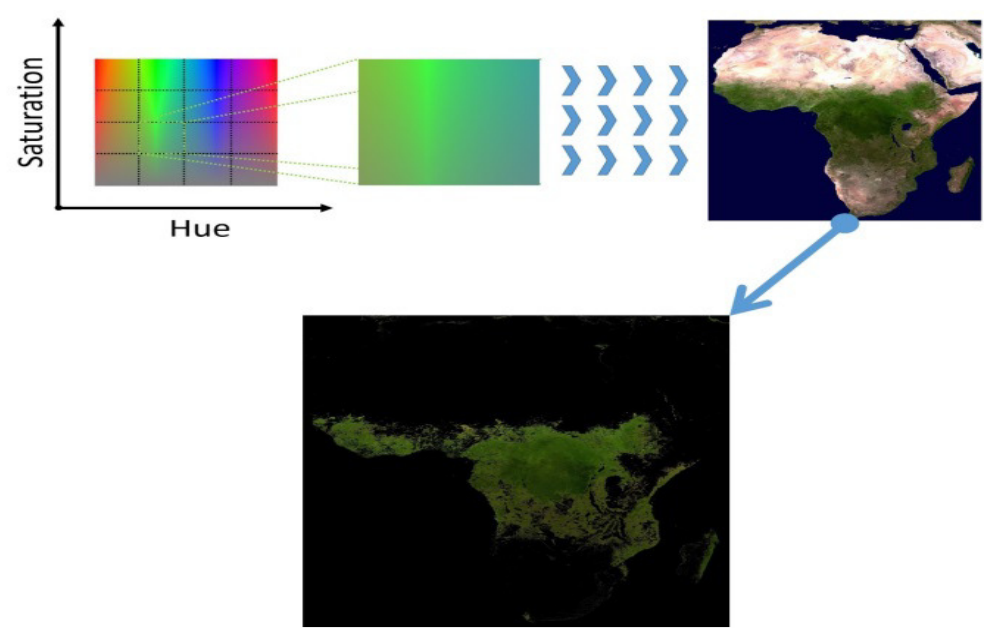

Figure .7 the output of masking a sample image with one sensitivity region

For each sensitivity level selection, a threshold is also selected for the acceptable area ratio for the clusters to ensure excluding the negligible clusters as a post-processing stage. This is important to only have clusters that actually represent something meaningful. When the sensitivity level is small, the threshold for the acceptable area ratio can have higher values than the threshold used when the sensitivity level is high because the number of colors included in small sensitivity regions is small, and therefore it is more likely to have small clusters which cannot be ignored. Figure 8 illustrates the cluster area ratios for eight clusters that represent the full area of an image including the negligible clusters. The clusters having an area ratio less than 5\% were neglected. 


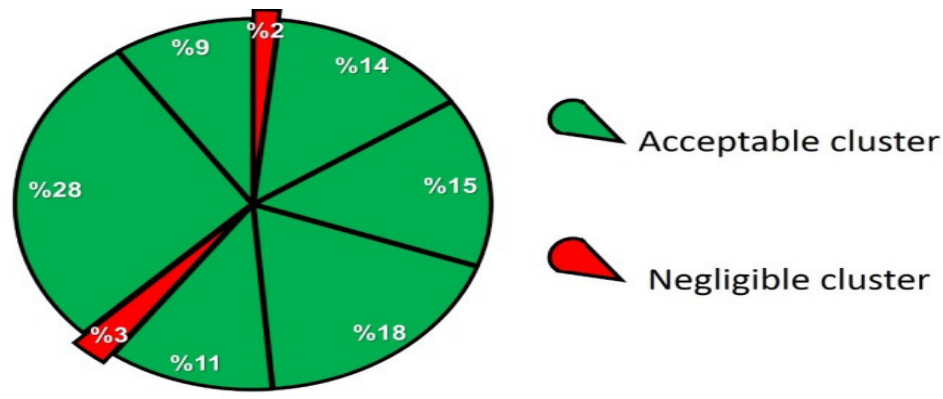

Figure 8.The cluster area ratios for eight clusters that represent the full area of an image including the negligible clusters

Figure 9 shows an example of the image resulting from the system after it finishes processing the input map image with the steps discussed above. The Sensitivity level used in the example is 1 , yielding 4 sensitivity regions. There was 1 negligible cluster when using a threshold for the acceptable cluster area ratio $10 \%$ with HSI color space.

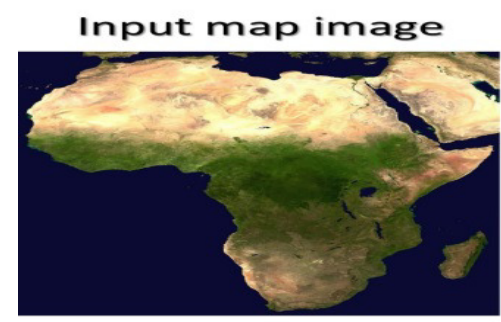

Cluster 2

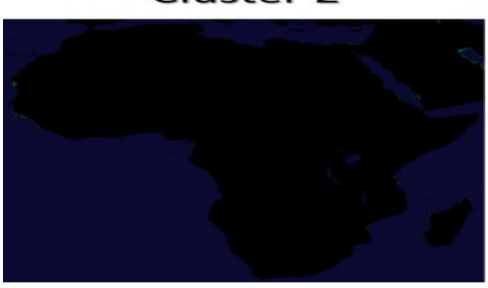

Figure 9. An example of map image clustering processed in the proposed system

\section{RESULTS ANALYSIS}

This part summarizes the experiments performed to evaluate the proposed map clustering system. The experiments investigate using two color spaces: HSI and L*a*b.

\subsection{QUALITATIVE EXPERIMENTS}

The qualitative evaluation performed in this research shows that the clustering results when using HIS color space is better than the results when using $\mathrm{L}^{*} \mathrm{a} * \mathrm{~b}$ color space, as shown in Figure 10. 


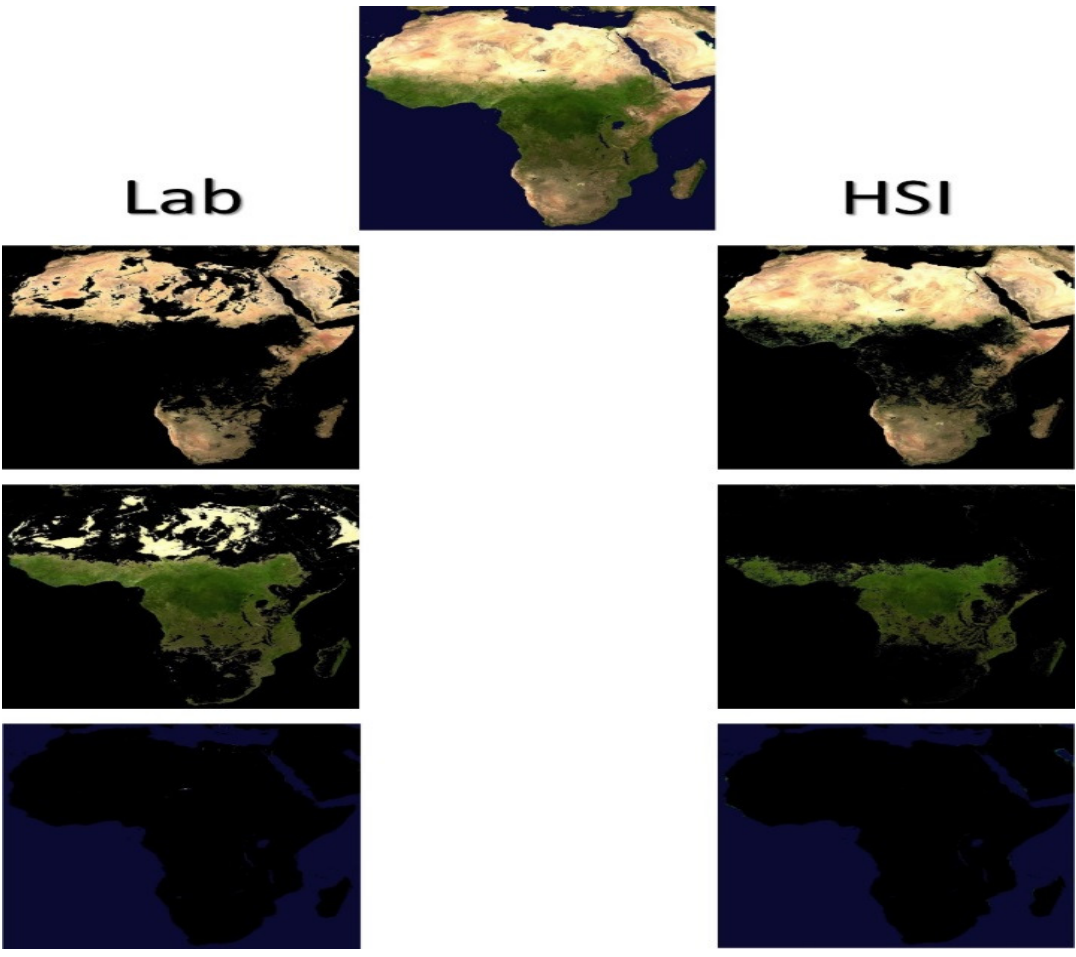

Figure 10. Map image clustering results when using HSI and $\mathrm{L} * \mathrm{a} * \mathrm{~b}$ color spaces

Figures 11 and 12 show examples of the Arab World Map ( includes 14 colors) resulting from the system after it finishes processing the input map image with the steps discussed above. The Sensitivity level used in the example is 4 , yielding 32 sensitivity regions. There were 14 negligible clusters when using a threshold for the acceptable cluster area ratio $01 \%$ using $\mathrm{L}^{*} \mathrm{a} * \mathrm{~b}$ color space. But when we use HSI colorspace there were 17 negligible clusters using the same sensitivity regions.

Classification based on HSI color system was more professional than $\mathrm{L}^{*} \mathrm{a} * \mathrm{~b}$, it could give real and exact clusters.

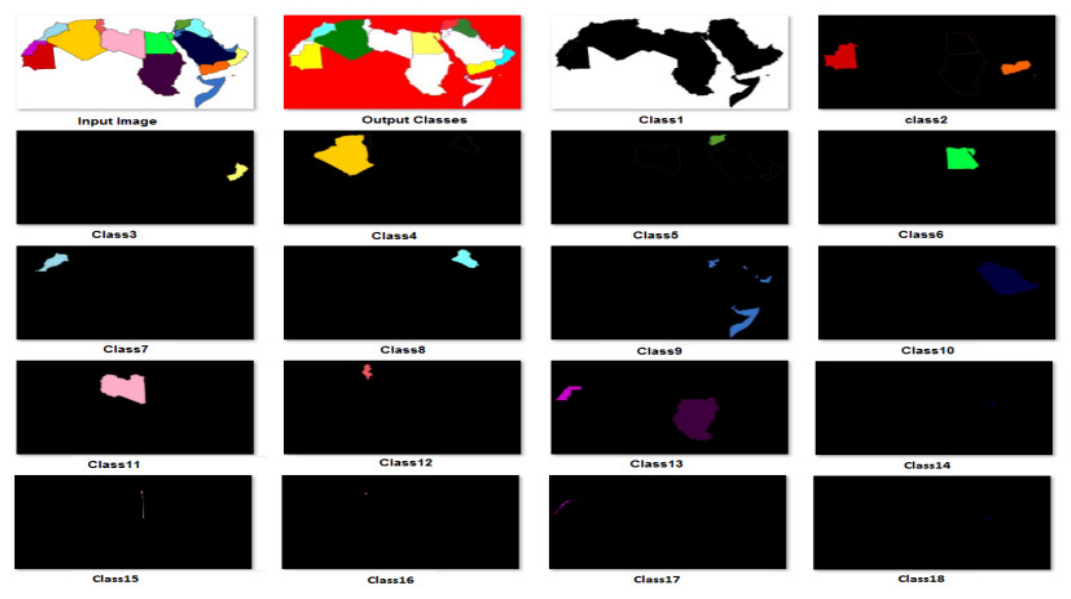

Figure 11. An example of the Arab World Map image clustering based on $\mathrm{L}^{*} \mathrm{a} * \mathrm{~b}$ color space. 

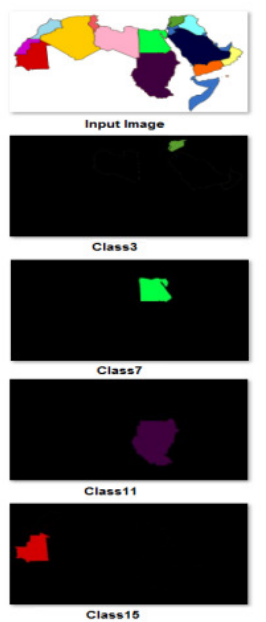
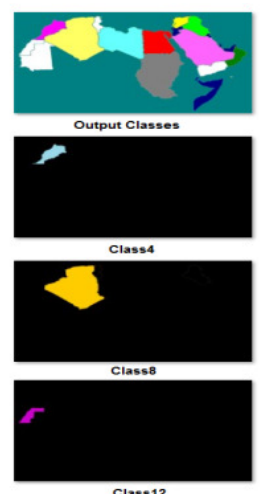

Class 12
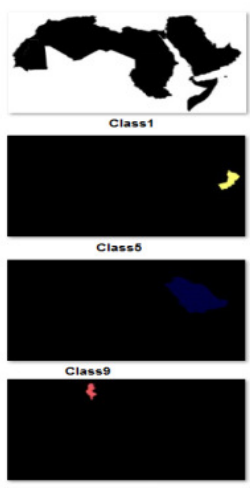

Class 13
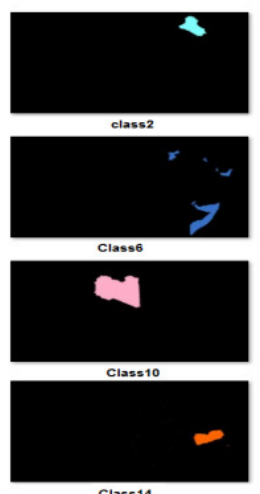

Class 14

Figure 12. An example of the Arab World Map image clustering based on HSI color space

\subsection{QUANTITATIVE EXPERIMENTS}

As previously discussed, the sensitivity level used in the system controls the number of clusters found in the image, based on Equation (2). Figure 13 shows the number of clusters in HSI and $\mathrm{L}^{*} \mathrm{a} * \mathrm{~b}$ color spaces with different negligible area ratios (NR).

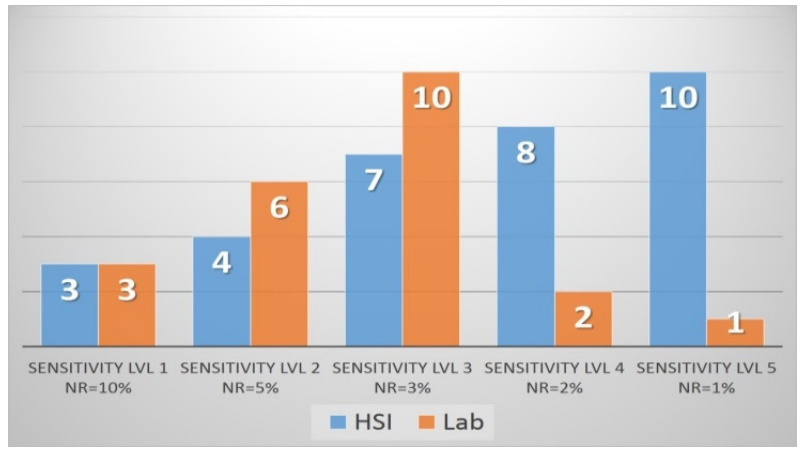

Figure 13. The number of clusters in HSI and $\mathrm{L}^{*} \mathrm{a} * \mathrm{~b}$ color spaces with different negligible area ratios (This graph indicates that the numbers of clusters when it was little)

The Figure shows that when the sensitivity level is increased, the number of clusters in HSI color space is increasing reasonably even though a negligible area ratio is used. However, after sensitivity level 3 (when using $L^{*} a^{*} b$ color space), the number of clusters decreases unexpectedly - it was expected to increase as what happens when the HSI color space is used. This huge unexpected decrease in the number of clusters is the result of dividing the image to meaningless clusters whereas the visuallysimilar regions on the map are divided into multiple clusters instead of one cluster and consequently, instead of one acceptable cluster, the area of one region will be divided to many clusters of which many are negligible.

Figure 14 shows that when the sensitivity level is increased, the number of clusters in HSI color space will increase. However, the increase in the number of clusters in $L^{*} a * b$ in sensitivity levels 3 - 4 was arbitrary and inaccurate . 
International Journal of Computer Networks \& Communications (IJCNC) Vol.8, No.3, May 2016

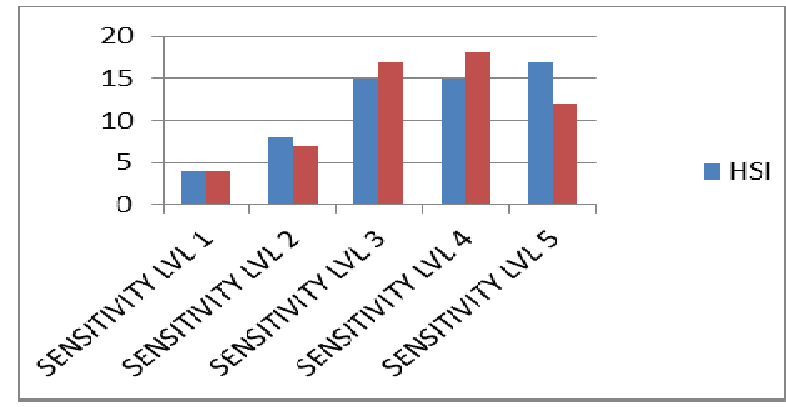

Figure 14. The number of clusters in HSI and $\mathrm{L}^{*} \mathrm{a} * \mathrm{~b}$ color spaces with different negligible area ratios (This graph indicates The numbers of clusters when it was 14)

The performance of the system when using HSI and $\mathrm{L}^{*} \mathrm{a} * \mathrm{~b}$ color spaces was also investigated. For this experiment, the time required to process an image of size 6.5 Mega-pixel is measured using the function timeit available on Mathworks. Time measurements have been performed in the following specifications:

- Windows 7 64-bit

- Intel(R) Core(TM) i7-2670QM CPU @ 2.20GHz

- 6M Cache

- 6144MB RAM

- MATLAB R2013a software package

Figure 15 shows the time required to complete the map image clustering in the proposed system when using HSI and $\mathrm{L}^{*} \mathrm{a} * \mathrm{~b}$ color spaces.

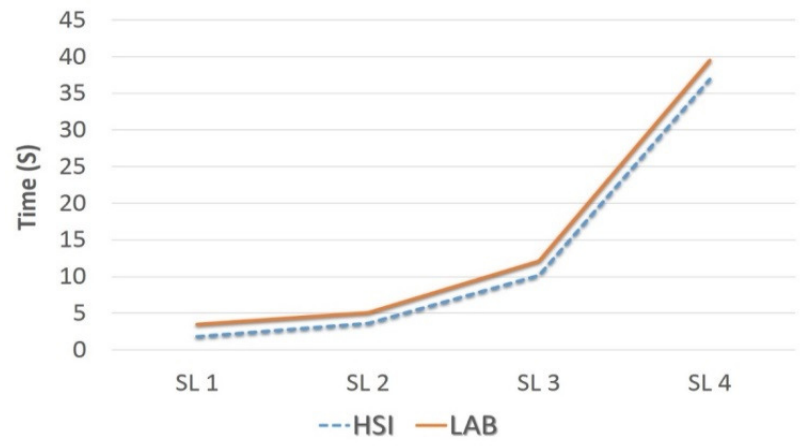

Figure 15.the time required to complete the map image clustering in the proposed system when using HSI and $\mathrm{L}^{*} \mathrm{a} * \mathrm{~b}$ color spaces.

The time required increases when the sensitivity level (SL) is increased because the number of masks used in clustering increases when the number of sensitivity regions increase, which means more processing is required. The difference in performance between the system when using HSI and when using $\mathrm{L}^{*} \mathrm{a} * \mathrm{~b}$ color spaces is small, with a mean difference equals to two seconds. The HSI color conversion is two seconds faster than $\mathrm{L}^{*} \mathrm{a}$ *b color conversion. 
International Journal of Computer Networks \& Communications (IJCNC) Vol.8, No.3, May 2016

\section{Conclusion}

This paper presented an effective maps clustering system, in which a map image is processed to categorize the natural regions in the map image into clusters, based on color. The system was implemented using a combination of several image processing techniques, such as image contrast enhancement, color space conversion and masking. The processed map image undergoes several stages to extract the clusters, including a preprocessing stage which makes the clustering process more accurate. Another addition proposed in the system to make it more effective, is sensitivity level which will be used in the system to define the number of equally-sized color regions for each cluster. Each region includes a set of color shades that will be mapped to a single cluster.

The presented research investigated the map images clustering system, evaluated the performance and compared the efficiency of using two color spaces: HSI and $\mathrm{L}^{*} \mathrm{a} * \mathrm{~b}$, using qualitative and quantitative experiments. The results of the qualitative experiments performed showed that the map image clustering results were better when using HSI than the when using $\mathrm{L}^{*} \mathrm{a} * \mathrm{~b}$ color space, as the clusters were more accurate.

In quantitative experiments, using HSI color space was found to have an increase in the number of clusters when increasing the sensitivity level. In other words, using HSI color space is more effective in maps clustering when high sensitivity is required, whereas using $\mathrm{L}^{*} \mathrm{a} * \mathrm{~b}$ color space yields many negligible clusters.

The time required to complete the map image clustering when using HSI and L*a*b color system when changing the sensitivity level was also compared. The time required increases when increasing sensitivity level. The required processing time when using HSI is smaller than when using $\mathrm{L} * \mathrm{a} * \mathrm{~b}$ color space.

To sum up, this research presented an effective map clustering system using image processing techniques. The system achieves accurate clustering when using HSI color space with a slightly better performance in the processing time needed.

\section{FUTURE WORK}

In addition to the research of the future, we aspire to the classification of geographical maps on other color systems and to comparing the results of this research in order to produce the best color classification model.

\section{REFERENCES}

[1] Al- ZoubiMoh'dBelal( 2010 ), A New Algorithm for Automatic Extraction of GIS Layers “,7th International Multi-Conference on Systems Signals and Devices (SSD), IEEE, Amman.

[2] Arum .S Sari and Suciati .N ( 2014 ), Flower Classification using Combined a* b* Color and Fractalbased Texture Feature “,Vol.7, No.2, pp.357-368, Surabaya, Indonesia : International Journal of Hybrid Information Technology.

[3] Atkinsona .P.M, Lewisb .P, (2000), Geostatistical Classification for Remote Sensing: An Introduction", Computers \&Geosciences vol. 26 , pp. 361-371.

[4] Berberoglua .S, Lloydb .C.D, Atkinsonb .P.M and Curranb .P.J, (2000), The Integration of Spectral and Textural Information Using Neural Networks for Land Cover Mapping in The Mediterranean, Computers \& Geosciences, vol. 26, pp. 385-396.

[5] Caprioli .M, and Tarantino .E, (2001), Accuracy Assessment of Per-Field Classification Integrating Very Fine SpatialResolution Satellite Imagery with Topographic Data, Journal of Geospatial Engineering, vol. 3, no. 2, pp. 127-134. 
[6] Farah .Y. A, Baki R. M, Yassin .A, Tahir.N and Ishak .W, (2009), Monitoring of Watermelon Ripeness Based on Fuzzy Logic, WRI World Congress on Computer Science and Information Engineering, pp.,67-70.

[7] Lei Xu (2011), A new method for license plate detection based on color and edge information of lab space. In: Proceedings of International Conference on Multimedia and Signal Processing, (CMSP'2011), p. 99- 102, USA: IEEE.

[8] Mena .J .B, (2003), State of the Art on Automatic Road Extraction for GIS Update: A Novel Classification, Pattern Recognition Letters, vol. 24, no. 16, pp. 3037-3058.

[9] Mena .J .B, Malpica .J .A, (2005), An Automatic Method For Road Extraction in Rural and SemiUrban Areas Starting from High Resolution Satellite Imagery, Pattern Recognition Letters, vol. 26, pp. 1201-1220,.

[10] Rongjie Liu, Zhang Jie, Song Pingjian, Shao Fengjing and Guanfeng Liu, (2008), An Agglomerative Hierarchical Clustering Based High-Resolution Remote Sensing Image Segmentation Algorithm, International Conference on Computer Science and Software Engineering, pp. 403-406.

[11] Russ .J.C ( 2011), The Image Processing Handbook, ISBN 9781439840450, US : CRC Press.

\section{Authors}

Salam M. Gandour, received a BSc degree in software engineering from the ISRA University Amman, Jordan, 2009 and an MSc in CIS from the University of Jordan, Amman in 2015. Her research interests include Geographic Maps eLearning and IoT.

Dr.Nidal Mahmmoud Mustafa Turab received a BSc degree in communication engineering from the University of Garounis, Benghazi, libya 1992 and an MSc in telecommunication engineering from the University of Jordan, Amman in 1996. His PhD in computer science is from the Polytechnic University of Bucharest, 2008. His research interests include WLAN security, computer network security and cloud computing security, eLearning and Artificial Intelligent. $\mathrm{He}$ is an associate professor at Alhliyya AmmanUniversity, Amman, Jordan 